\title{
Prótesis unicompartimental lateral de rodilla en el tratamiento del genu valgo artrósico. Resultados en 29 artroplastias con un seguimiento promedio de 6.2 años
}

\author{
Gabriel Gaggiotti," Stefano Gaggiotti,", Julio C. Ringa" \\ "COT - Centro de Ortopedia y Traumatología Rafaela y Santa Fe, Argentina \\ **Servicio de Ortopedia y Traumatología, Hospital Británico de Buenos Aires, Ciudad Autónoma de Buenos Aires, Argentina
}

\begin{abstract}
RESUMEN
Objetivo: Evaluar, de manera retrospectiva, los resultados funcionales y la supervivencia a mediano plazo de la prótesis unicompartimental lateral de rodilla para tratar el genu valgo artrósico. Materiales y Métodos: Estudio observacional retrospectivo. Se analizaron los casos operados con prótesis unicompartimental lateral de rodilla por genu valgo artrósico entre enero de 1999 y enero de 2019, seguimiento mínimo de un año. Se evaluaron los resultados clínicos y funcionales mediante el KSS 2011, el grado de artrosis en el compartimento externo y su progresión en el compartimento contralateral con la clasificación de Kellgren y Lawrence, y la condropatía femororrotuliana mediante la clasificación de Outerbridge modificada. Se determinaron la incidencia de complicaciones y la supervivencia de la prótesis. Resultados: Se evaluaron 29 prótesis unicompartimentales laterales de rodilla en 27 pacientes, con un seguimiento promedio de 6.2 años. El KSS clínico y funcional se incrementó de 56,5 $\pm 9,8$ a 91,9 $\pm 5,3$ y de $33,9 \pm 13,7$ a $91,4 \pm 10,3$, respectivamente, $(p<0,001)$. La flexión máxima mejoró de 106, $6^{\circ} \pm 6,7^{\circ}$ a $124,2^{\circ} \pm 2,4^{\circ}$ y la contractura en flexión, de $5,2^{\circ} \pm 3,2^{\circ}$ a $1^{\circ} \pm 1,6^{\circ}$ ( $\left.p<0,001\right)$. El eje preoperatorio fue de $12,3^{\circ} \pm 4,1^{\circ}$ de valgo, para un posoperatorio de $5,2^{\circ} \pm 3,1^{\circ}$ de valgo ( $p<0,001$ ). La supervivencia de la prótesis fue del $100 \%$, con un caso de progresión artrósica en el compartimento medial $(3,4 \%)$. Conclusión: La prótesis unicompartimental lateral de rodilla representa una alternativa válida y definitiva para tratar la patología artrósica femorotibial externa.
\end{abstract}

Palabras clave: Prótesis unicompartimental; lateral; genu valgo artrósico; artroplastia de rodilla; reemplazo unicompartimental. Nivel de Evidencia: IV

Lateral Unicompartmental Knee Arthroplasty for the Treatment of Lateral Knee Osteoarthritis.

Results in 29 Arthroplasties with an Average Follow-up of 6.2 Years

\section{ABSTRACT}

Objective: The purpose of this article is to examine the medium-term functional outcomes and survivorship of lateral unicompartmental knee arthroplasty in the treatment of lateral knee osteoarthritis.Materials and Methods: Retrospective report. We selected and analysed all patients who had undergone a lateral unicompartmental knee arthroplasty for the treatment of lateral knee osteoarthritis between January 1999 and January 2019, with a minimum follow-up of 1 year. The KSS score system 2011, the Kellgren-Lawrence osteoarthritis classification, the Outerbridge femoropatellar chondropathy classification and serial radiographs were used in the evaluation of each patient. The complication and prosthesis survivorship rates were assessed. Results: We identified 29 lateral unicompartmental knee arthroplasties in 27 patients with a follow-up of 6.2 years (1-19.5). The clinical and functional KSS improved from $56.5 \pm 9.8$ to $91.9 \pm 5.3$ and $33.9 \pm 13.7$ to $91.4 \pm 10.3$ respectively $(p<0.001$ ). Postoperative maximal flexion improved from $106^{\circ} \pm 6.7^{\circ}$ to $124.2^{\circ} \pm 2.4^{\circ}$ and flexion contracture improved from $5.2^{\circ} \pm 3.2^{\circ}$ to $1^{\circ} \pm 1.6^{\circ}(p<0.001)$. The average preoperative alignment was $12.3^{\circ} \pm 4.1^{\circ}$ of valgus angulation, which was corrected to $5.2^{\circ} \pm 3.1^{\circ}$ of valgus $(p<0.001)$. The

Recibido el 27-9-2020. Aceptado luego de la evaluación el 8-12-2020 • Dr. GABRIEL GAGGIOTTI • gabrielgaggiotti@ hotmail.com (ID https://orcid.org/0000-0001-7839-4597 Cómo citar este artículo: Gaggiotti G, Gaggiotti S, Ringa JC. Prótesis unicompartimental lateral de rodilla en el tratamiento del genu valgo artrósico. Resultados en 29 artroplastias con un seguimiento promedio de 6.2 años. Rev Asoc Argent Ortop Traumatol 2021;86(3):299-308. https://doi.org/10.15417/issn. 1852-7434.2021.86.3.1211 
survivorship rate was $100 \%$ and only one patient showed osteoarthritic changes in the medial compartment (3.4\%). Conclusion: Lateral unicompartmental knee arthroplasty provides excellent medium-term results. It represents a reliable and definitive option in the treatment of the isolated lateral knee osteoarthritis.

Keywords: Unicompartmental knee arthroplasty; lateral; lateral knee osteoarthritis; knee arthroplasty; unicompartmental replacement.

Level of Evidence: IV

\section{INTRODUCCIÓN}

La prótesis unicompartimental de rodilla (PUC) surgió en la década de 1970 como una alternativa terapéutica en pacientes con artrosis femorotibial interna o externa aislada. ${ }^{1}$ Inicialmente su empleo fue controvertido a raíz de los resultados poco satisfactorios y las elevadas tasas de revisión. ${ }^{2}$ No obstante, desde la década de 1980, varios autores, como Cartier y cols. en particular, difundieron ampliamente su uso, perfeccionando la técnica, las indicaciones y el diseño de la prótesis. ${ }^{3,4}$ En la actualidad, su utilización se está incrementando en una relación de 3 a 1 respecto de la prótesis total de rodilla (PTR). ${ }^{5}$

La PUC representa una alternativa atractiva y menos invasiva, con preservación del stock óseo, cartilaginoso, ligamentario y de propioceptividad, con menos sangrado operatorio, menor riesgo de infección y costos económicos más bajos en relación con la PTR. Otras ventajas son el mayor rango de movilidad obtenido en el posoperatorio, el menor tiempo de recuperación y de baja laboral, con un patrón de marcha y una cinemática articular más fisiológicos. ${ }^{6,7}$ Además, se trata de un procedimiento definitivo en la gran mayoría de los casos, con tasas de supervivencia superiores al $90 \%$ a 10 años. ${ }^{3,4,8,9}$

En 1984, Marmor publicó el primer estudio focalizado en la PUC lateral, con resultados excelentes en un seguimiento promedio de 89 meses. ${ }^{10} \mathrm{La}$ artrosis femorotibial externa aislada es menos frecuente que en el compartimento interno, tiene una incidencia del 5-10\% de las rodillas artrósicas. ${ }^{11,12}$ La PUC lateral es 10 veces menos frecuente que la PUC medial, representa menos del 1\% de todas las artroplastias. Además de su menor prevalencia, la PUC lateral es técnicamente más demandante y menos reproducible, por la biomecánica propia y más compleja del compartimento externo. ${ }^{11,12}$

El objetivo de este estudio fue evaluar los resultados funcionales y la supervivencia a mediano plazo de la PUC lateral en el tratamiento del genu valgo artrósico. Nuestra hipótesis es que la PUC lateral tiene resultados funcionales y una supervivencia similares a los publicados en la bibliografía internacional.

\section{MATERIALES Y MÉTODOS}

Se llevó a cabo un estudio observacional retrospectivo para evaluar los resultados funcionales y la supervivencia a mediano plazo de la PUC lateral en el tratamiento del genu valgo artrósico. Se analizaron los casos operados, de forma consecutiva, por el mismo cirujano, con la misma técnica, entre enero de 1999 y enero de 2019.

Los criterios de inclusión fueron: 1) pacientes con genu valgo artrósico que cumplen con las indicaciones para una PUC lateral, 2) edad $>18$ años, 3) seguimiento $>12$ meses. Los criterios de exclusión fueron: 1) pacientes con genu valgo artrósico tratado con PTR por no cumplir con indicaciones de PUC, 2) pérdida de seguimiento.

\section{Evaluación clínica}

Los datos preoperatorios se obtuvieron, en forma retrospectiva, mediante la revisión de las historias clínicas de los pacientes que cumplieron los criterios de inclusión. La valoración clínica se realizó antes de la cirugía y en el último control posoperatorio mediante la escala del Knee Society Scoring System (KSS) 2011. La estabilidad articular se constató mediante maniobras de bostezo varo-valgo, Lachman, pivot-shift y cajón anteroposterior, y la movilidad articular se evaluó con un goniómetro. Durante la intervención, se valoraron la condropatía femororrotuliana según la clasificación de Outerbridge modificada y la integridad de los ligamentos cruzados anterior (LCA) y posterior.

En los controles posoperatorios, se evaluó si había complicaciones tanto agudas (antes de los 3 meses) como tardías. Se consideró revisión a cualquier nueva intervención quirúrgica realizada en la rodilla operada, consistente en el retiro o recambio de alguno de los componentes protésicos, y reintervención a aquellas con conservación de los componentes. 


\section{Evaluación radiográfica}

Antes de la cirugía, se tomaron radiografías de ambas rodillas, de frente y de perfil con carga bipodálica, axial de rótula a $30^{\circ}$ de flexión (Merchant), de frente en semiflexión de $45^{\circ}$ (Schuss), y radiografías en varo y valgo forzado para evaluar la suficiencia de los ligamentos colaterales, la corrección del deseje y el pinzamiento del compartimento contralateral (Figura 1). En el posoperatorio, se tomaron radiografías de rótula, de frente, de perfil y axial.

Se midió el eje femorotibial con un goniómetro antes de la operación y después de ella. Se cuantificó el grado de artrosis en el compartimento externo y la existencia de progresión en el compartimento contralateral según la escala de Kellgren y Lawrence. Las evaluaciones estuvieron a cargo de uno de los autores que no intervino en la cirugía.
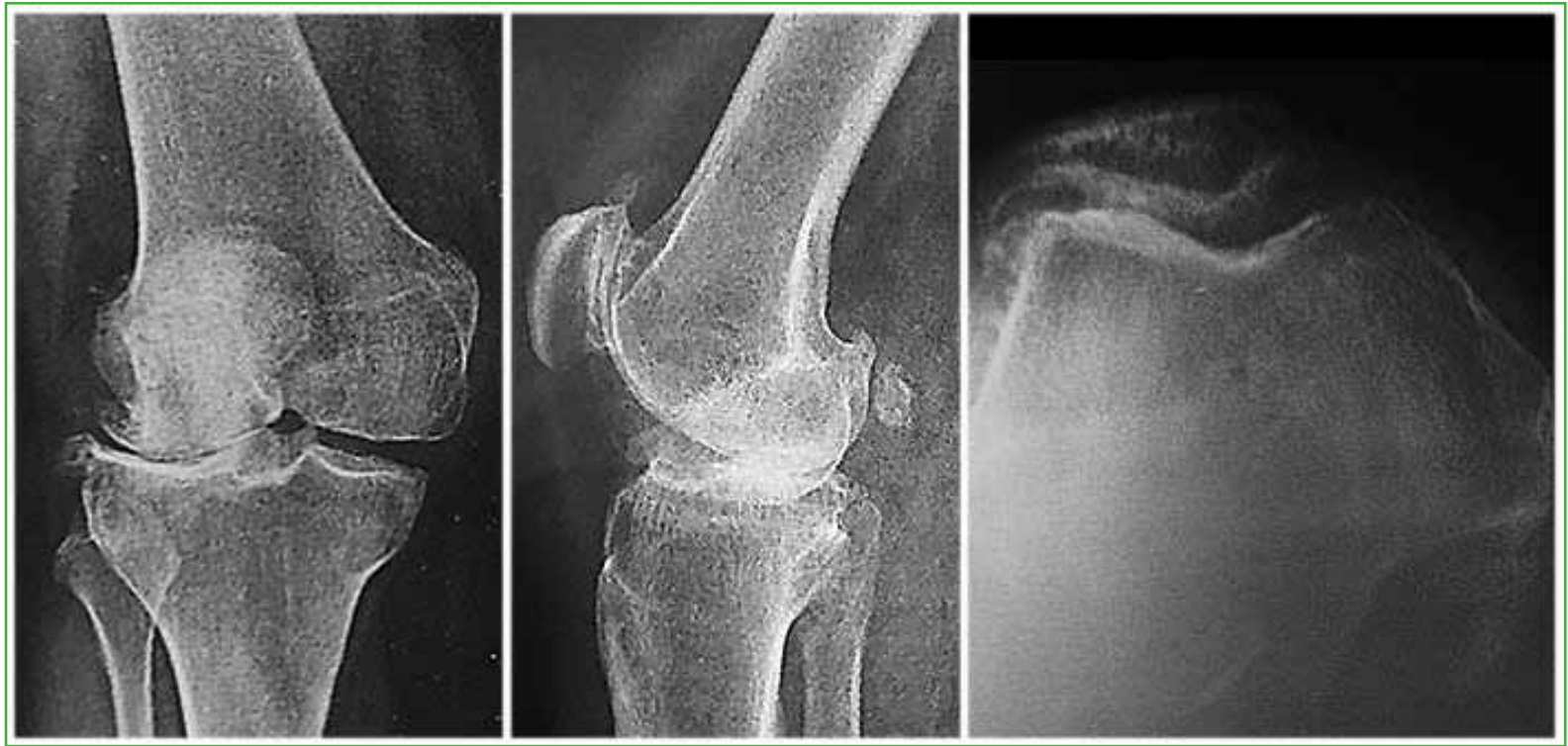

Figura 1. Radiografías de rodilla derecha preoperatorias. Genu valgo artrósico grado 4 de Kellgren y Lawrence con artrosis femororrotuliana.

\section{Indicaciones}

La PUC se indicó por un genu valgo artrósico sintomático confirmado clínica y radiográficamente, dolor localizado en la interlínea articular externa asociado a cambios artrósicos en el compartimento lateral, deformidad corregible en las radiografías con estrés en varo, con conservación del espacio articular en el compartimento medial; deseje en valgo de hasta $20^{\circ}$, flexión preoperatoria $>90^{\circ}$, déficit de extensión preoperatorio $<15^{\circ}$ e índice de masa corporal $\leq 35$; suficiencia ligamentaria clínica en los planos coronal y sagital.

Indicaciones extendidas: no consideramos contraindicaciones a los cambios artrósicos sintomáticos o asintomáticos a nivel femororrotuliano, osteofitos o artrosis incipiente sin repercusión clínica en el compartimento medial, lesión degenerativa del LCA sin inestabilidad clínica secundaria a la progresión artrósica, como tampoco la edad del paciente en el momento de la cirugía. Las artropatías inflamatorias, como artritis reumatoide, en pacientes $<65$ años con enfermedad inactiva, bajo tratamiento médico y buen stock óseo, no se consideraron una contraindicación.

Contraindicaciones: genu valgo artrósico con compromiso bicompartimental femorotibial, pinzamiento del compartimento medial en las radiografías de rodilla con estrés en varo, desejes fijos en valgo o valgo severo $>20^{\circ}$, flexión preoperatoria $<90^{\circ}$, flexo preoperatoria $>15^{\circ}$, índice de masa corporal $>35$, inestabilidad clínica anteropos-terior o mediolateral y artropatías sistémicas activas. 


\section{Técnica quirúrgica}

Paciente en decúbito dorsal bajo anestesia raquídea. Incisión convencional reducida en línea media y abordaje pararrotuliano externo puro trans-retinacular sin afectación del tendón cuadricipital, con desplazamiento de la rótula en sentido medial sin eversión. Se respetan los osteofitos laterales del cóndilo femoral externo que sirven de apoyo al componente femoral. Resección de osteofitos tibiales anteriores, condilares posteriores y en escotadura intercondílea (notchplastia) para liberar el LCA, si fuera necesario, y facilitar la recuperación del flexo preoperatorio.

Con respecto a los cortes óseos tibial y femoral, se debe lograr el estricto paralelismo en extensión entre el corte femoral distal y el corte tibial horizontal, a fin de alcanzar una alineación y un centrado correctos entre ambos componentes en la posición de la marcha. Para esto, el componente tibial debe localizarse lo más medial posible, sin lesionar con el corte la inserción del LCA ni el tendón rotuliano, el cual es medializado con un separador. El componente femoral se debe posicionar hiperlateralizado con la rodilla en flexión. En flexión de rodilla, los componentes tibial y femoral tienen una orientación divergente, mientras que se alinean con la extensión, debido al movimiento de atornillamiento femorotibial (Figura 2).

Se debe lograr una laxitud de seguridad de $2-3 \mathrm{~mm}$ o bostezo al varo $(+/++)$ en flexión de $20-30^{\circ}$, a fin de evitar una hipercorrección que lleve al deterioro del compartimento contralateral. Es de vital importancia conservar la integridad del ligamento colateral lateral y el tendón poplíteo, ya que su lesión favorece la hipercorrección de la deformidad por inestabilidad lateral. En todos los casos, se utilizó una prótesis unicompartimental de platillo fijo. No se empleó intensificador de imágenes.

Sobre la articulación femororrotuliana, se realizan los siguientes gestos quirúrgicos a demanda: resección de osteofitos, shaving cartilaginoso, microfracturas, facetectomía externa rotuliana o prótesis femororrotuliana. Finalmente, se procede al cierre subsinovial dejando abierto el alerón externo a fin de disminuir la hiperpresión externa femororrotuliana y lograr un encarrilado rotuliano adecuado.

Alta hospitalaria entre las 24 y 36 h posteriores a la cirugía, con carga completa, ejercicios isométricos de cuádriceps y profilaxis antitrombótica por vía oral, durante cuatro semanas. Fisiokinesioterapia a partir de las 3-4 semanas y retorno a las actividades habituales a las 6-8 semanas.
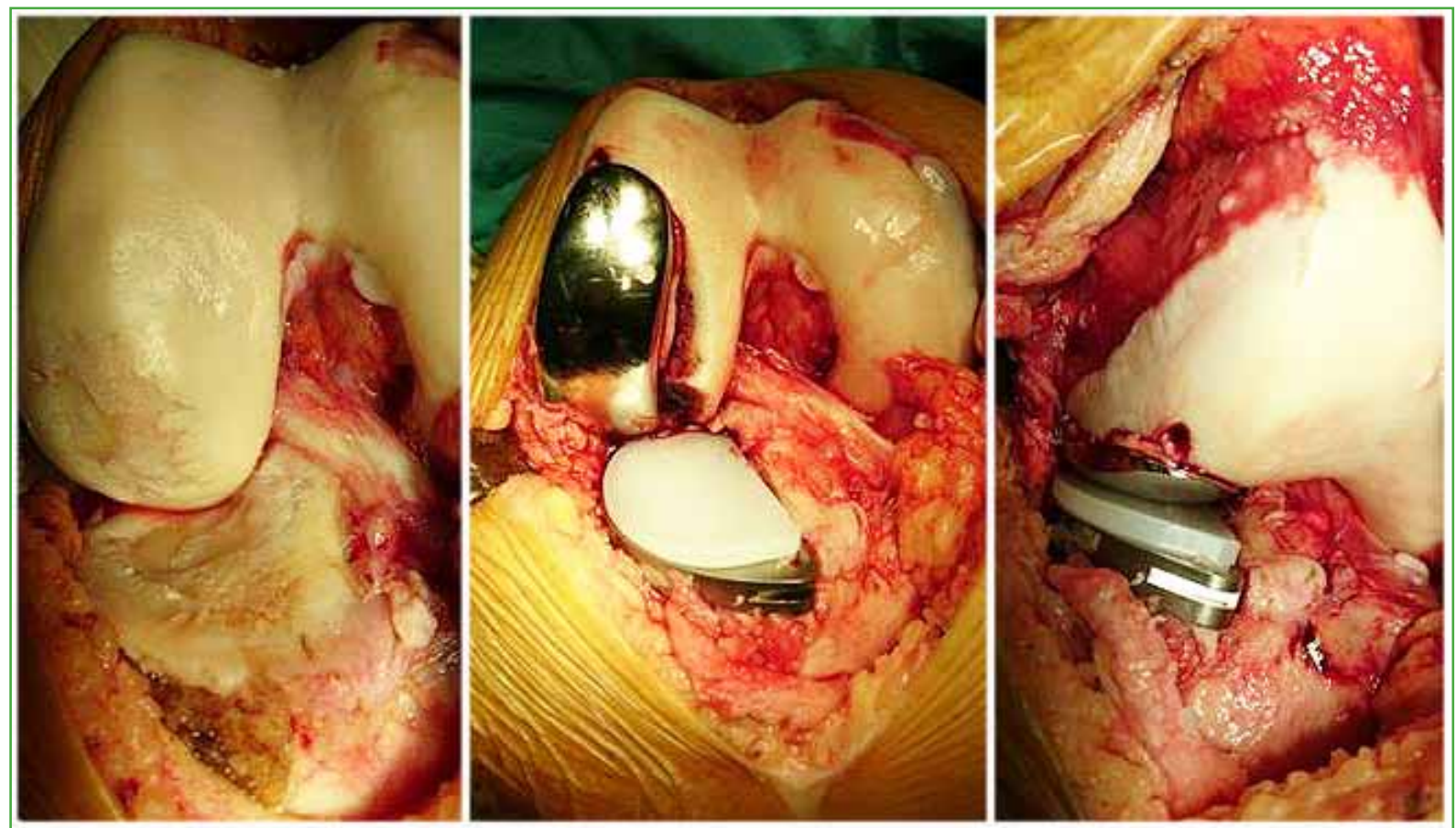

Figura 2. Fenómeno de "atornillamiento" femorotibial: divergencia de los componentes de la prótesis en flexión y convergencia en extensión o posición de la marcha. 


\section{Análisis estadístico}

Los datos recolectados se volcaron en una planilla de Microsoft Excel para su posterior análisis con los programas R Studio y Tableau Desktop. Los resultados se expresan en tablas de frecuencia o gráficos, según corresponda. Para las variables cuantitativas se utilizaron histogramas y se calcularon las medidas de posición (media, mediana y cuantiles) y las medidas de dispersión (desviación estándar y el rango intercuartílico) correspondientes. Las pruebas de hipótesis se realizaron considerando un nivel de significación del 5\% y las pruebas utilizadas se escogieron en función de la naturaleza de los datos. Para comparar las características de los individuos pretratamiento y postratamiento, se emplearon la prueba t o el test de rangos de Wilcoxon, en ambos se trabaja con su adaptación para muestras pareadas. Para estudiar la correlación entre variables se utilizó la prueba de correlación no paramétrica de Spearman.

\section{RESULTADOS}

De una serie de 304 PUC, 33 fueron laterales en 31 pacientes. Se excluyó a cuatro pacientes por fallecimiento no relacionado con la cirugía, con pérdida del seguimiento. La muestra quedó conformada por 29 PUC laterales por genu valgo artrósico en 27 pacientes, con un seguimiento promedio de 6.2 años (rango 1-19.5). En dos casos, el procedimiento se llevó a cabo de forma bilateral y simultánea en un mismo tiempo quirúrgico. Tres pacientes tenían cirugías previas (artroscopia). Las características demográficas se exponen en la Tabla 1.

Tabla 1. Características demográficas de la muestra

\begin{tabular}{|l|c|}
\hline $\begin{array}{l}\text { Total de pacientes } \\
\text { Total de prótesis unicompartimentales de rodilla laterales }\end{array}$ & 27 \\
\hline Edad (años) & 29 \\
\hline Índice de masa corporal & 64.3 (rango 50-80) \\
\hline Seguimiento (años) & 28,6 (rango 25,4-33,8) \\
\hline $\begin{array}{l}\text { Sexo } \\
\text { Masculino }\end{array}$ & 6.2 (rango 1-19.5) \\
\hline $\begin{array}{l}\text { Femenino } \\
\text { Causas } \\
\text { Gonartrosis primaria }\end{array}$ & $8(25,9 \%)$ \\
\hline Osteonecrosis del cóndilo femoral externo & $21(74,1 \%)$ \\
\hline
\end{tabular}

Los implante utilizados fueron: 23 prótesis ZUK (Zimmer®, Warsaw, IN, EE.UU.), cuatro Allegretto (Sulzer, Winterthur, Suiza) y dos MG (Zimmer®, Warsaw, IN, EE.UU.).

La condropatía femororrotuliana intraoperatoria según la clasificación de Outerbridge modificada fue de grado 4 ( 10 rodillas), grado 3 (13 rodillas) y grado 2 (6 rodillas). En cuatro de los pacientes con condropatía grado 4, que presentaban subluxación externa y pinzamiento femororrotuliano franco asociados, se practicó una facetectomía externa. Se detectó la ausencia del LCA en dos casos de valgo severo por progresión de la osteofitosis intercondílea, sin inestabilidad clínica.

Evaluación radiográfica preoperatoria femorotibial: todos los casos correspondieron al estadio 4 en la clasificación de Kellgren y Lawrence en el compartimento externo; se observaron dos casos de subluxación en el plano coronal. El eje preoperatorio fue de $12,3^{\circ} \pm 4,1^{\circ}$ de valgo, se destacaban 10 casos de valgo $>15^{\circ}$, entre ellos, tres casos severos de $20^{\circ}$, reductibles en maniobras de varo-valgo forzado. Evaluación radiográfica posoperatoria femorotibial: el eje fue de $5,2^{\circ} \pm 3,1^{\circ}$ de valgo $(\mathrm{p}<0,001)$ (Figura 3 ). Se detectó la progresión del proceso degenerativo artrósico en el compartimento medial en un paciente, que desarrolló cambios grado 2 y sintomatología interna. 


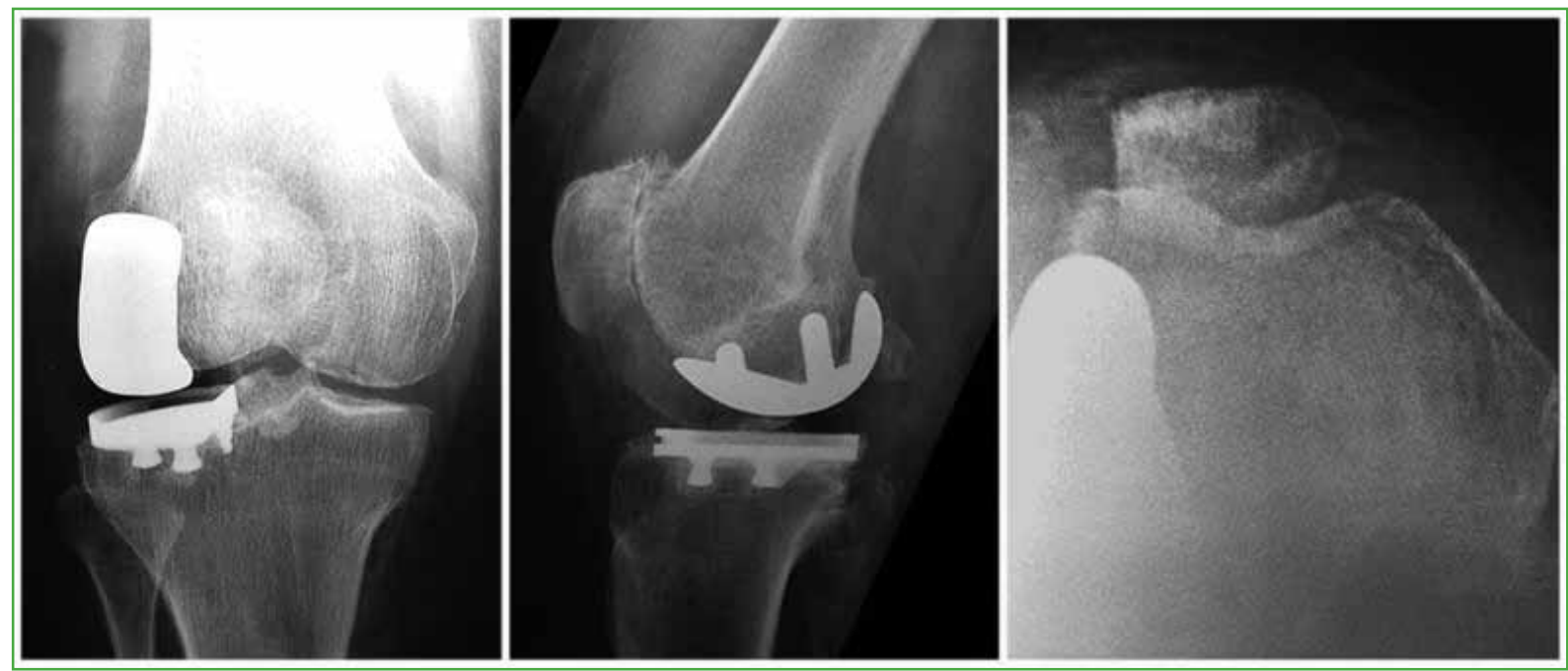

Figura 3. Radiografías de rodilla derecha posoperatorias. Correcta alineación de la prótesis y facetectomía rotuliana externa.

La mejoría del KSS fue estadísticamente significativa en todos los casos ( $p<0,001)$. El KSS clínico se incrementó de 56,5 \pm 9,8 antes de la cirugía a 91,9 \pm 5,3 después de ella y el KSS funcional fue de 33,9 $\pm 13,7$ y 91,4 $\pm 10,3$, respectivamente. Según el KSS, la satisfacción se incrementó de $12,7 \pm 4,6$ a 38,2 $\pm 3,6$ y las expectativas pasaron de 12,4 $\pm 1,4$ a 14,9 $\pm 0,6$. Se observó una mejoría estadísticamente significativa de la flexión máxima de $106,6^{\circ} \pm 6,7^{\circ}$ a $124,1^{\circ} \pm 2,4^{\circ}(p<0,001)$ y de la contractura en flexión de $5,2^{\circ} \pm 3,2^{\circ}$ a $1^{\circ} \pm 1,6^{\circ}(p<0,001)$ en el último control posoperatorio (Tabla 2).

Tabla 2. Resultados comparativos pre y posoperatorios

\begin{tabular}{|l|c|c|c|}
\hline & Preoperatorio & Posoperatorio & p \\
\hline KKS clínico & $56,5 \pm 9,8$ & $91,9 \pm 5,3$ & $<0,001$ \\
\hline KSS funcional & $33,9 \pm 13,7$ & $91,4 \pm 10,3$ & $<0,001$ \\
\hline KSS satisfacción & $12,7 \pm 4,6$ & $38,2 \pm 3,6$ & $<0,001$ \\
\hline KSS expectativas & $12,4 \pm 1,4$ & $14,9 \pm 0,6$ & $<0,001$ \\
\hline Flexión máxima & $106,6^{\circ} \pm 6,7^{\circ}$ & $124,1^{\circ} \pm 2,4^{\circ}$ & $<0,001$ \\
\hline Contractura en flexión & $5,2^{\circ} \pm 3,2^{\circ}$ & $1^{\circ} \pm 1,6^{\circ}$ & $<0,001$ \\
\hline Eje femorotibial & $12,3^{\circ} \pm 4,1^{\circ}$ & $5,2^{\circ} \pm 3,1^{\circ}$ & $<0,001$ \\
\hline
\end{tabular}

KSS $=$ Knee Society Score

Los resultados funcionales fueron similares para los procedimientos uni o bilaterales y para los diferentes implantes utilizados (Figura 4). Se observó una correlación negativa estadísticamente significativa entre la edad y el KSS funcional posoperatorio $(\mathrm{p}=0,04)$, y entre el índice de masa corporal y el KSS clínico posoperatorio $(\mathrm{p}=$ 0,006). No se observó una relación estadísticamente significativa entre el KSS posoperatorio y los grados de valgo o condropatía femororrotuliana según Outerbridge $(\mathrm{p}>0,05)$.

La supervivencia de la prótesis fue del $100 \%$ con un seguimiento de 6.2 años (rango 1-19.5). Se produjo una complicación tardía, con progresión artrósica en el compartimento interno, a los 4 años y 6 meses de la cirugía. Antes el paciente había sido sometido a una menisectomía parcial artroscópica por otro profesional, a los 3 años y 6 meses de la PUC lateral. De esta manera, la tasa de reintervención fue del 3,4\%, sin revisiones hasta el momento. 


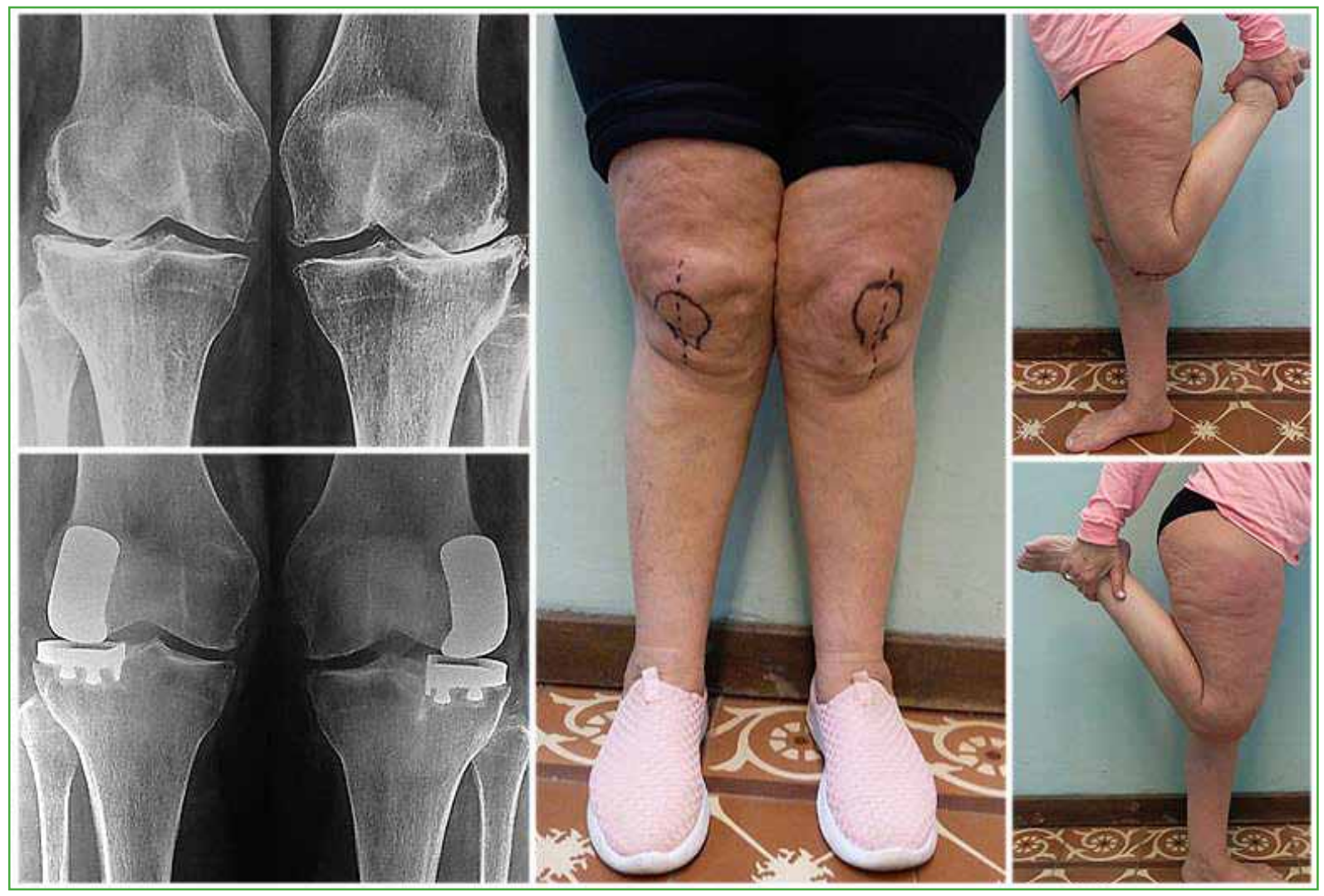

Figura 4. Paciente de 62 años con genu valgo bilateral tratado con prótesis unicompartimental de rodilla lateral bilateral en un tiempo, con 7 años de seguimiento. Correcta alineación de los componentes de la prótesis, con restauración del valgo primitivo y función completa de las rodillas.

\section{DISCUSIÓN}

El uso de la PUC medial o lateral en el tratamiento de la artrosis unicompartimental de rodilla fue controvertido en décadas pasadas, pero actualmente su uso está en franco aumento debido a los buenos resultados comunicados. ${ }^{5}$ La supervivencia a mediano plazo es comparable con la de la PTR, mientras que los resultados clínicos y funcionales son superiores. ${ }^{4,6-9,13-15}$ La menor frecuencia de la PUC lateral con respecto a la PUC medial (1:10) puede estar relacionada con la incidencia más baja de artrosis femorotibial externa aislada, como con las características biomecánicas propias de este compartimento que lo convierten en un procedimiento técnicamente más demandante. ${ }^{8,11,12}$

El progreso en el conocimiento clínico, la técnica quirúrgica y el diseño de la prótesis amplió las indicaciones clásicas y restrictivas de la PUC, definidas por Kozinn y Scott. ${ }^{15,16}$ La artrosis femororrotuliana, la edad, la obesidad, el nivel de actividad y la integridad de LCA dejaron de ser una contraindicación absoluta. ${ }^{15}$ Hamilton y cols., en su estudio retrospectivo de 1000 PUC mediales, no hallaron diferencias en las tasas de fracasos y reintervención entre aquellos pacientes que cumplían o no los criterios de selección clásicos. ${ }^{16}$ Comunicaron mejores resultados funcionales en el grupo donde el procedimiento hubiera estado contraindicado, el cual representaba el 68\% de la muestra. ${ }^{16}$ En nuestro estudio, dos pacientes tenían ausencia degenerativa del LCA sin inestabilidad preoperatoria; ocho, un índice de masa corporal de entre 30 y 35; y 10, condropatía grado 4 a nivel femororrotuliano. Tras la liberación del alerón rotuliano externo, el shaving condral, las microfracturas, la pateloplastia y la facetectomía externa, todos refirieron una mejoría clínica femororrotuliana. Además, tras el seguimiento, 10 pacientes <60 años no tuvieron signos de aflojamiento de la prótesis y solo uno de ellos tuvo una progresión artrósica del compartimento interno. De esta manera, consideramos que la lesión degenerativa del LCA sin inestabilidad clínica, el índice de masa corporal de entre 30 y 35 , el compromiso femororrotuliano y la edad $<60$ años antes de la cirugía no representan una contraindicación absoluta para la PUC lateral. 
Nuestra serie de 29 rodillas y un seguimiento de 6.2 años muestra resultados clínicos y funcionales según el KSS comparables con los publicados (Tabla 3). Nuestro KSS clínico fue de 91,9 \pm 5,3 y el funcional, de 91,4 $\pm 10,3$, tal como Berend y cols., y Lustig y cols., e incluso superiores a otros reportes, como los de Sah y Scott y Argenson y cols. ${ }^{9,13,17,18}$ Asimismo, la tasa de satisfacción ascendió hasta 38,2 $\pm 3,6$, lo que revela un alto grado de conformidad. Observamos una gran mejoría del rango de movilidad, que alcanzó una flexión máxima de $124,1^{\circ} \pm 2,4^{\circ}$, similar a lo publicado por Berend y cols., Argenson y cols., y Lustig y cols., ${ }^{9,13,17}$ En nuestro estudio, la tasa de supervivencia de la prótesis fue del $100 \%$ al final del seguimiento, tal como lo reportaron Pennington y cols., en su serie de 29 pacientes tras un promedio de 12.4 años. ${ }^{8}$ En la serie de O’Rourke y cols., tras 25 años de evaluación, la mayor publicada, la supervivencia fue del $72 \%$ en 14 PUC laterales. ${ }^{14}$

Tabla 3. Resultados comparativos con la bibliografía publicada

\begin{tabular}{|c|c|c|c|c|}
\hline Estudio & n & Seguimiento (años) & Resultados & Supervivencia \\
\hline $\operatorname{Marmor}^{10}(1984)$ & 13 & 7.4 (rango 2.3-9.8) & 11 excelentes & $92,3 \%$ (1 revisión) \\
\hline Ohdera y cols. ${ }^{19}$ (2001) & 18 & 8.3 (rango 5-15.7) & $89 \%$ buenos y excelentes (HSS) & $89 \%$ (2 revisiones) \\
\hline Ashraf y cols. ${ }^{21}(2002)$ & 83 & 9 (rango 2-21) & $\begin{array}{c}\text { BKS } 53.2 \text { preop., } 90.1 \text { a } 2 \text { años, } \\
83 \text { a } 10 \text { años }\end{array}$ & $\begin{array}{l}83 \% \text { a } 10 \text { años } \\
74,5 \% \text { a } 15 \text { años }\end{array}$ \\
\hline Pennington y cols. ${ }^{8}$ (2006) & 29 & 12.4 (rango 3.1-15.6) & $100 \%$ buenos y excelentes (HSS) & $100 \%$ \\
\hline Sah y Scott (2007) & 48 & 5.2 (rango $2-15)$ & KSS clínico 89 y funcional 80 & $100 \%$ \\
\hline Argenson y cols. ${ }^{9}(2008)$ & 38 & 12.6 (rango $3-23)$ & KSS clínico 88 y funcional 78 & $\begin{array}{l}92 \% \text { a } 10 \text { años } \\
84 \% \text { a } 16 \text { años }\end{array}$ \\
\hline Berend y cols. ${ }^{13}$ (2012) & 100 & 3.25 (rango 2-6.8) & KSS clínico 94 y funcional 89 & $97 \%$ \\
\hline Lustig y cols. ${ }^{17}$ (2014) & 46 & 14.2 (rango 10.2-18) & KSS clínico 95 y funcional 82 & $\begin{array}{l}94,4 \% \text { a } 10 \text { años } \\
91,4 \% \text { a } 15 \text { años }\end{array}$ \\
\hline Edmiston y cols. ${ }^{20}$ (2018) & 65 & 6.8 (mínimo 2) & KSS combinado 146 & $94 \%$ \\
\hline Nuestra serie (2020) & 29 & 6.2 (rango 1-19.5) & KSS clínico 92 y funcional 91 & $100 \%$ \\
\hline
\end{tabular}

HSS $=$ Hospital for Special Surgery knee score. BKS = Bristol Knee Score.

La tasa de complicaciones fue del 3,4\%. Hubo una complicación tardía que requirió de una intervención artroscópica a cargo de otro profesional a los 3 años y 6 meses de la PUC lateral, con menisectomía parcial interna por sintomatología medial. El último control posoperatorio mostró la progresión artrósica en el compartimento interno, lo cual podría atribuirse a una hipercorrección. De esta manera, la tasa libre de reintervención fue del 96,5\%. La hipocorrección del eje es la regla de oro para evitar el deterioro del compartimento contralateral por sobrecarga durante la fase estática y dinámica de la marcha, con el objetivo de restaurar el eje primitivo del miembro. ${ }^{3,11,12}$ En nuestra serie, de manera similar a lo publicado, el eje femorotibial posoperatorio fue $5,2^{\circ} \pm 3,1^{\circ}$ de valgo. ${ }^{17,19}$

La progresión artrósica en el compartimento opuesto es poco frecuente según los reportes bibliográficos, en la mayoría de los casos es asintomática. ${ }^{89.20}$ Sin embargo, representa la principal causa de revisión protésica, la cual puede realizarse mediante la conversión a una PTR o utilizando una PUC medial. ${ }^{17}$ Según la bibliografía y nuestra experiencia en revisiones de PUC mediales, en la mayoría de los casos de conversión a PTR, esta puede realizarse de forma relativamente sencilla, y es necesario usar vástagos e implantes de revisión en un tercio de los casos. ${ }^{21-23}$

Debido al diferente radio de curvatura entre ambos cóndilos femorales, el fenómeno de rollback durante la flexión de rodilla se da fundamentalmente en el compartimento externo. Numerosos estudios biomecánicos confirmaron este movimiento de rotación externa del fémur e interno de la tibia durante la flexión, con posterior rotación externa tibial asociada a rotación interna femoral durante la extensión, dando lugar al mecanismo de atornillamiento o "screw-home mechanism" que bloquea la rodilla en extensión. ${ }^{24}$ Esta discrepancia en el rollback femoral ayuda a explicar la incongruencia que habitualmente se observa en la alineación de los componentes durante la flexión, que se corrige en extensión o posición de la marcha al realizar la PUC lateral. ${ }^{12}$ 
Debido al fenómeno de atornillamiento, los implantes de platillo tibial móvil no son una alternativa válida para el compartimento femorotibial externo, a causa de su elevada tasa de luxación. En su serie de 53 PUC laterales realizadas con prótesis Oxford de platillo móvil, Gunther y cols. informaron una supervivencia de la prótesis del $82 \%$ a los 5 años de seguimiento, con 11 casos de revisión y un $10 \%$ de luxación del inserto de polietileno. ${ }^{25}$ En concordancia con la bibliografía, nuestra serie sugiere que las PUC de platillo fijo se pueden utilizar, de manera segura, en el compartimento externo, con resultados satisfactorios y predecibles. ${ }^{8,9,13}$

Este estudio presenta las limitaciones inherentes a la metodología observacional y retrospectiva. Como debilidades destacamos el número relativamente escaso de pacientes que conforman la muestra, lo cual puede explicar la baja frecuencia de complicaciones. Sin embargo, no encontramos publicaciones nacionales similares y las publicaciones internacionales no muestran, en general, un número amplio de pacientes, por lo que creemos que nuestro análisis puede contribuir a la bibliografía de nuestro medio. Dentro de las fortalezas, es importante remarcar que la población del estudio fue homogénea. Además, todos los pacientes fueron operados por el mismo equipo quirúrgico, con un mismo tipo de implante y realizaron el mismo protocolo de rehabilitación. Son necesarios estudios con una muestra más amplia y un seguimiento más prolongado.

\section{CONCLUSIONES}

La PUC lateral representa una alternativa válida y definitiva en el tratamiento de la patología artrósica femorotibial externa. El carácter conservador del procedimiento, la calidad de los resultados funcionales, la rápida recuperación y las escasas complicaciones, el mejor costo-beneficio, más una correcta indicación y una técnica quirúrgica rigurosa, hacen de la PUC lateral el procedimiento de elección para un número creciente de cirujanos. Hay que tener en cuenta que se trata de un procedimiento técnicamente demandante con una curva de aprendizaje más prolongada en relación con la PUC medial.

Conflicto de intereses: El doctor Gabriel Gaggiotti realiza actividades de cirujano Consultor para Zimmer Biomet.

El resto de los autores no declara conflictos de intereses.

ORCID de S. Gaggiotti: https://orcid.org/0000-0003-4077-2892

ORCID de J. C. Ringa: https://orcid.org/0000-0002-8137-2337

\section{BIBLIOGRAFÍA}

1. Marmor L. Marmor modular knee in unicompartmental disease. Minimum four-year follow-up. J Bone Joint Surg Am 1979;61(3):347-53. https://doi.org/10.2106/00004623-197961030-00005

2. Insall J, Aglietti P. A five to seven-year follow-up of unicondylar arthroplasty. J Bone Joint Surg Am 1980;62(8): 1329-37. https://doi.org/10.2106/00004623-198062080-00013

3. Cartier P, Gaggiotti G, Jully JL. Primary osteonecrosis of the medial femoral condyle. Unicompartmental or total replacement? Int Orthop 1988;12(3):229-35. https://doi.org/10.1007/BF00547168

4. Cartier P, Sanouiller JL, Grelsamer RP. Unicompartmental knee arthroplasty surgery. 10-year minimum follow-up period. J Arthroplasty 1996;11(7):782-8. https://doi.org/10.1016/s0883-5403(96)80177-x

5. Riddle DL, Jiranek WA, McGlynn FJ. Yearly incidence of unicompartmental knee arthroplasty in the United States. J Arthroplasty 2008;23(3):408-12. https://doi.org/10.1016/j.arth.2007.04.012

6. Kim MS, Koh IJ, Choi YJ, Lee JY, In Y. Differences in patient-reported outcomes between unicompartmental and total knee arthroplasties: A propensity score-matched analysis. J Arthroplasty 2017;32(5):1453-9. https://doi.org/10.1016/j.arth.2016.11.034

7. Robertsson O, Borgquist L, Knutson K, Lewold S, Lidgren L. Use of unicompartmental instead of tricompartmental prostheses for unicompartmental arthrosis in the knee is a cost-effective alternative. 15,437 primary tricompartmental prostheses were compared with 10,624 primary medial or lateral unicompartmental pros. Acta Orthop Scand 1999;70(2):170-5. https://doi.org/10.3109/17453679909011257 
8. Pennington DW, Swienckowski JJ, Lutes WB, Drake GN. Lateral unicompartmental knee arthroplasty: Survivorship and technical considerations at an average follow-up of 12.4 years. $J$ Arthroplasty 2006;21(1):13-7. https://doi.org/10.1016/j.arth.2004.11.021

9. Argenson JNA, Parratte S, Bertani A, Flecher X, Aubaniac JM. Long-term results with a lateral unicondylar replacement. Clin Orthop Relat Res 2008;466(11):2686-93. https://doi.org/10.1007/s11999-008-0351-Z

10. Marmor L. Lateral compartment arthroplasty of the knee. Clin Orthop Relat Res 1984;186(4):115-21. https://doi.org/10.1097/00003086-198406000-00019

11. Ollivier M, Abdel MP, Parratte S, Argenson JN. Lateral unicondylar knee arthroplasty (UKA): Contemporary indications, surgical technique, and results. Int Orthop 2014;38(2):449-55. https://doi.org/10.1007/s00264-013-2222-9

12. Servien E, Merini A, Lustig S, Neyret P. Lateral uni-compartmental knee replacement: Current concepts and future directions. Knee Surg Sport Traumatol Arthrosc 2013;21(11):2501-8. https://doi.org/10.1007/s00167-013-2585-x

13. Berend KR, Kolczun MC, George JW, Lombardi A V. Lateral unicompartmental knee arthroplasty through a lateral parapatellar approach has high early survivorship. Clin Orthop Relat Res 2012;470(1):77-83. https://doi.org/10.1007/s11999-011-2005-9

14. O’Rourke MR, Gardner JJ, Callaghan JJ, Liu SS, Goetz DD, Vittetoe DA, et al. Unicompartmental knee replacement: A minimum twenty-one-year followup, end-result study. Clin Orthop Relat Res 2005;(440):27-37. https://doi.org/10.1097/01.blo.0000185451.96987.aa

15. Heyse TJ, Khefacha A, Peersman G, Cartier P. Survivorship of UKA in the middle-aged. Knee 2012;19(5):585-91. https://doi.org/10.1016/j.knee.2011.09.002

16. Hamilton TW, Pandit HG, Jenkins C, Mellon SJ, Dodd CAF, Murray DW. Evidence-based indications for mobile-bearing unicompartmental knee arthroplasty in a consecutive cohort of thousand knees. J Arthroplasty 2017;32(6):1779-85. https://doi.org/10.1016/j.arth.2016.12.036

17. Lustig S, Lording T, Frank F, Debette C, Servien E, Neyret P. Progression of medial osteoarthritis and long term results of lateral unicompartmental arthroplasty: 10 to 18 year follow-up of 54 consecutive implants. Knee 2014;21(1):26-32. https://doi.org/10.1016/S0968-0160(14)50006-3

18. Sah AP, Scott RD. Lateral unicompartmental knee arthroplasty through a medial approach: Study with an average five-year follow-up. J Bone Joint Surg Am 2007;89(9):1948-54. https://doi.org/10.2106/JBJS.F.01457

19. Ohdera T, Tokunaga J, Kobayashi A. Unicompartmental knee arthroplasty for lateral gonarthrosis: Midterm results. J Arthroplasty 2001;16(2):196-200. https://doi.org/10.1054/arth.2001.2090

20. Edmiston TA, Manista GC, Courtney PM, Sporer SM, Della Valle CJ, Levine BR. Clinical outcomes and survivorship of lateral unicompartmental knee arthroplasty: Does surgical approach matter? J Arthroplasty 2018;33(2):362-5. https://doi.org/10.1016/j.arth.2017.09.009

21. Ashraf T, Newman JH, Evans RL, Ackroyd CE. Lateral unicompartmental knee replacement survivorship and clinical experience over 21 years. J Bone Joint Surg Br 2002;84(8):1126-30. https://doi.org/10.1302/0301-620x.84b8.13447

22. Thienpont E. Conversion of a unicompartmental knee arthroplasty to a total knee arthroplasty can we achieve a primary result? Bone Joint J 2017;99B(1):65-9. https://doi.org/10.1302/0301-620X.99B1.BJJ-2016-0272

23. Leta TH, Lygre SHL, Skredderstuen A, Hallan G, Gjertsen JE, Rokne B, et al. Outcomes of unicompartmental knee arthroplasty after aseptic revision to total knee arthroplasty a comparative study of 768 TKAs and 578 UKAs revised to TKAs from the norwegian arthroplasty register (1994 to 2011). J Bone Joint Surg Am 2016;98(6):431-40. https://doi.org/10.2106/JBJS.O.00499

24. Pinskerova V, Johal P, Nakagawa S, Sosna A, Williams A, Gedroyc W, et al. Does the femur roll-back with flexion? J Bone Joint Surg Br 2004;86(6):925-31. https://doi.org/10.1302/0301-620x.86b6.14589

25. Gunther TV, Murray DW, Miller R, Wallace DA, Carr AJ, O'Connor JJ, et al. Lateral unicompartmental arthroplasty with the Oxford meniscal knee. Knee 1996;3(1-2):33-9. https://doi.org/10.1016/0968-0160(96)00208-6 